https://doi.org/10.15407/ujpe64.2.109

V.I. ROMANENKO,${ }^{1}$ N.V. KORNILOVSKA,${ }^{2}$ O.G. UDOVYTSKA,${ }^{1}$ L.P. YATSENKO ${ }^{1}$

${ }^{1}$ Institute of Physics, Nat. Acad. of Sci. of Ukraine

(46, Nauky Ave., Kyiv 03680,Ukraine; e-mail: vr@iop.kiev.ua)

2 Kherson National Technical University

(24, Berislavske Highway, 73008 Kherson, Ukraine)

\title{
SPATIAL DISTRIBUTION OF ATOMS IN THE FIELD OF INTERSECTING STANDING BICHROMATIC LIGHT WAVES
}

\begin{abstract}
We have shown that, by properly detuning the carrier frequencies in each of two perpendicularly intersecting bichromatic waves from the atomic transition frequency, it is possible to create a two-dimensional trap for atoms, if the wave intensities are sufficiently high. At the zero and near-zero values of the initial wave phases, as well as at the phase shift between the intersecting waves equal to $\pi$ or close to $\pi$ values, the dynamic spatial patterns of atoms consisting of square cells with the side length equal to $\lambda / \sqrt{2}$ are formed. Numerical simulations were carried out for sodium atoms.

Keywords: optical atomic trap, standing waves, Monte Carlo wave function approach.
\end{abstract}

\section{Introduction}

Various aspects of the interaction between atoms with standing bichromatic waves - this is a pair of monochromatic standing waves with different frequencies, which can also be considered as counterpropagating bichromatic waves or counter-propagating amplitude-modulated waves - have been studied for three decades. The physical fundamentals of this interaction and numerous experimental works were analyzed, in particular, in books $[1,2]$ and in the recent review [3].

The force of light pressure on an atom in the field of a standing bichromatic wave can significantly exceed that of a running monochromatic wave [4-9], and this fact is essential for the efficient manipulation with atomic beams [9-11]. The field of bichromatic waves was found to be applicable to cool down atoms and molecules making no use of spontaneous emission [12, 13]. This is a new phenomenon, which was predicted

(C) V.I. ROMANENKO, N.V. KORNILOVSKA,

O.G. UDOVYTSKA, L.P. YATSENKO, 2019

ISSN 2071-0194. Ukr. J. Phys. 2019. Vol. 64, No. 2 in work [14]. Seemingly, it can strongly extend our abilities to control the molecular motion with the help of laser radiation.

Another direction of researches includes the formation of atomic traps making use of only laser radiation without additional fields (e.g., the magnetic field in the case of magneto-optical trap [1]). At first, such traps were proposed to be based on the interaction of atoms with counter-propagating light pulses [15-18]. Later, it was found that required traps can also be formed by counter-propagating bichromatic [19], stochastic [20], and frequency modulated [21] waves. Furthermore, the laser radiation field that holds atoms in the trap can cool them down as well.

In this work, we consider the interaction of twolevel atoms with two orthogonal plane-polarized standing bichromatic waves. We analyze the conditions, under which those waves can form a two-dimensional atomic trap (the one-dimensional trap has been studied by us in work [19]), and determine the parameters of the patterns formed by atoms at their interaction with the light wave field. The motion of 

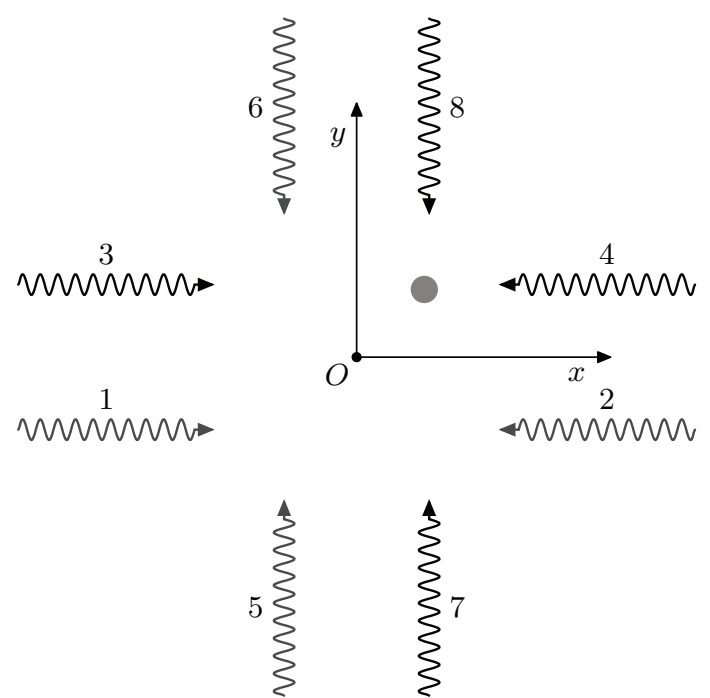

Fig. 1. Schematic diagram of the interaction between the atom (denoted by the circle) and the field. At point $O$, the phase difference between the counter-propagating waves equals zero

atoms in the field is described by Newton's laws, and the evolution of atomic states by the Monte Carlo wave-function method [22]. The numerical simulation is carried out for parameters corresponding to the interaction of sodium atoms with the light field.

The structure of the present paper is as follows. In the next section, a scheme of the interaction between an atom and the field is described. The basic equations are written down in Section 3. A brief description of the Monte Carlo wave-function method is made in Section 4. Section 5 is devoted to the procedure of numerical calculations, whereas the results obtained are presented and their discussion is made in Section 6. Short conclusions are formulated in the final section of the paper.

\section{Interaction of an Atom with the Field}

Let an atom be in the field of two perpendicularly intersecting bichromatic standing waves (see Fig. 1). Each of the latter is formed by a pair of standing collinear waves. In turn, each of the standing waves can be regarded as two counter-propagating monochromatic waves.

We consider the interaction of atoms with the bichromatic field of standing waves near point $O$, where each standing wave has an antinode, i.e. the phase difference of the corresponding counter-propagating waves equals zero. In the field of one bichromatic wave, if an atom becomes slightly shifted from this point, it undergoes an action of a light pressure force that is proportional to the phase difference between the standing waves that form this bichromatic wave $[4,23]$. Since the phase difference linearly depends on the atomic coordinates, a bichromatic standing light wave can form a one-dimensional trap for atoms $[6,19]$. Hence, a field of two intersecting bichromatic waves could expectedly form a two-dimensional atomic trap.

Note that the interaction of an atom with two perpendicularly intersecting standing bichromatic waves is qualitatively different from the interaction with one wave. Besides the absorption from either of the running bichromatic waves and the subsequent emission into the counter-propagating running bichromatic wave, there can occur the absorption from either of the running bichromatic wave and the subsequent emission into the orthogonally propagating running bichromatic wave. Therefore, the interaction between the atom and the field created by two standing bichromatic waves cannot be regarded as the sum of interactions of this atom with each of the standing bichromatic waves.

\section{Basic Equations}

The interaction of an atom with the field of intersecting bichromatic waves can also be considered as its interaction with the field of eight running monochromatic waves, as is shown in Fig. 1. The space-time dependences of the field intensities created by the running waves look like

$\mathbf{E}_{n=1,2,3,4}=\mathbf{e} E_{0 n} \cos \left(\omega_{n} t \mp k_{n} x+\varphi_{n}\right)$,

$\mathbf{E}_{n=5,6,7,8}=\mathbf{e} E_{0 n} \cos \left(\omega_{n} t \mp k_{n} y+\varphi_{n}\right)$.

Here, the sign "_" corresponds to odd and the sign "+" to even $n$-values, $\mathbf{e}$ is the unit polarization vector, and $\omega_{n}$ is the monochromatic wave frequency. It is worth to note that the polarization vector directions were chosen to be identical for all waves. For eight indicated monochromatic waves to form four standing waves, the following condition has to be obeyed:

$\omega_{2 n}=\omega_{2 n-1}, \quad(n=1 \div 4)$.

In the considered case of formation of standing waves with identical amplitudes,

$E_{0 n}=E_{0}, \quad(n=1 \div 8)$. 
The detunings $\delta_{n}$ of the wave frequencies $\omega_{n}(n=$ $=1 \div 8$ ) from the resonance with the atomic transition frequency $\omega_{0}$ are defined as the differences

$\delta_{n}=\omega_{0}-\omega_{n}$,

for which

$\delta_{2 n-1}=\delta_{2 n}, \quad(n=1 \div 4)$,

because the frequencies of the counter-propagating monochromatic waves that form each of four standing waves are identical [see Eq. (3)]. Let the modulation frequencies of bichromatic waves, which are equal to the frequency difference between the running monochromatic waves, be also identical,

$\omega_{1}-\omega_{3}=\omega_{5}-\omega_{7}=\Omega>0$.

Here, we consider a symmetric case where the carrier frequencies of intersecting waves are also identical, i.e.

$\frac{\omega_{3}+\omega_{1}}{2}=\frac{\omega_{7}+\omega_{5}}{2}=\omega$

From the above equations, it is easy to express the frequencies of all monochromatic waves in terms of the bichromatic wave carrier frequency $\omega$ and the frequency of their modulation $\Omega$ :

$\omega_{1}=\omega_{5}=\omega+\frac{\Omega}{2}, \quad \omega_{3}=\omega_{7}=\omega-\frac{\Omega}{2}$.

Let us introduce the detuning

$\delta=\omega_{0}-\omega$

of the bichromatic wave carrier frequency from the atomic transition frequency. Then, in view of Eq. (9), Eq. (5) implies that

$\delta_{1}=\delta_{5}=\delta-\frac{\Omega}{2}, \quad \delta_{3}=\delta_{7}=\delta+\frac{\Omega}{2}$.

The field created by eight running monochromatic waves can be written as a field of four standing waves. The field strengths of the latter look like

$$
\begin{aligned}
& \mathbf{E}_{12}=\mathbf{E}_{1}+\mathbf{E}_{2}=2 E_{0} \mathbf{e} \cos \left[\omega_{1} t+\frac{1}{2}\left(\varphi_{1}+\varphi_{2}\right)\right] \times \\
& \times \cos \left[k_{1} x+\frac{1}{2}\left(\varphi_{2}-\varphi_{1}\right)\right]
\end{aligned}
$$

$$
\begin{aligned}
& \mathbf{E}_{34}=\mathbf{E}_{3}+\mathbf{E}_{4}=2 E_{0} \mathbf{e} \cos \left[\omega_{3} t+\frac{1}{2}\left(\varphi_{3}+\varphi_{4}\right)\right] \times \\
& \times \cos \left[k_{3} x+\frac{1}{2}\left(\varphi_{4}-\varphi_{3}\right)\right], \\
& \mathbf{E}_{56}=\mathbf{E}_{5}+\mathbf{E}_{6}=2 E_{0} \mathbf{e} \cos \left[\omega_{1} t+\frac{1}{2}\left(\varphi_{5}+\varphi_{6}\right)\right] \times \\
& \times \cos \left[k_{1} y+\frac{1}{2}\left(\varphi_{6}-\varphi_{5}\right)\right], \\
& \mathbf{E}_{78}=\mathbf{E}_{3}+\mathbf{E}_{4}=2 E_{0} \mathbf{e} \cos \left[\omega_{3} t+\frac{1}{2}\left(\varphi_{7}+\varphi_{8}\right)\right] \times \\
& \times \cos \left[k_{3} y+\frac{1}{2}\left(\varphi_{8}-\varphi_{7}\right)\right],
\end{aligned}
$$

and the total field acting on the atom equals

$\mathbf{E}=\mathbf{E}_{12}+\mathbf{E}_{34}+\mathbf{E}_{56}+\mathbf{E}_{78}$

Starting from the early studies of the mechanical action of bichromatic wave fields on atoms $[4,6]$, those fields are also presented as a superposition of counter-propagating amplitude-modulated waves, which allows an analogy between the bichromatic wave field and the field created by a sequence of counter-propagating pulses to be made. This analogy forms a basis for the explanation of the induced light pressure force. The latter can substantially exceed the force of light pressure on the atom by a single running wave [24]. In the case of two intersecting standing waves, the counter-propagating waves look like

$$
\begin{aligned}
& \mathbf{E}_{13}=\mathbf{E}_{1}+\mathbf{E}_{3}=2 E_{0} \mathbf{e} \cos \left[\omega t-k x+\frac{1}{2}\left(\varphi_{1}+\varphi_{3}\right)\right] \times \\
& \times \cos \left[\frac{1}{2} \Omega t-\frac{1}{2} \Delta k x+\frac{1}{2}\left(\varphi_{3}-\varphi_{1}\right)\right],
\end{aligned}
$$

$\mathbf{E}_{24}=\mathbf{E}_{2}+\mathbf{E}_{4}=2 E_{0} \mathbf{e} \cos \left[\omega t+k x+\frac{1}{2}\left(\varphi_{2}+\varphi_{4}\right)\right] \times$

$\times \cos \left[\frac{1}{2} \Omega t+\frac{1}{2} \Delta k x+\frac{1}{2}\left(\varphi_{4}-\varphi_{2}\right)\right]$,

$\mathbf{E}_{57}=\mathbf{E}_{1}+\mathbf{E}_{3}=2 E_{0} \mathbf{e} \cos \left[\omega t-k y+\frac{1}{2}\left(\varphi_{5}+\varphi_{7}\right)\right] \times$

$\times \cos \left[\frac{1}{2} \Omega t-\frac{1}{2} \Delta k y+\frac{1}{2}\left(\varphi_{7}-\varphi_{5}\right)\right]$,

$\mathbf{E}_{68}=\mathbf{E}_{2}+\mathbf{E}_{4}=2 E_{0} \mathbf{e} \cos \left[\omega t+k y+\frac{1}{2}\left(\varphi_{6}+\varphi_{8}\right)\right] \times$

$\times \cos \left[\frac{1}{2} \Omega t+\frac{1}{2} \Delta k y+\frac{1}{2}\left(\varphi_{8}-\varphi_{6}\right)\right]$,

ISSN 2071-0194. Ukr. J. Phys. 2019. Vol. 64, No. 2 
where

$k=\frac{1}{2}\left(k_{1}+k_{3}\right)=\frac{1}{2}\left(k_{2}+k_{4}\right)$,

$\Delta k=k_{3}-k_{1}=k_{4}-k_{2}=\Omega / c$.

The shift of the time $t$ and the coordinates $(x, y)$ of the reference starting point is obviously equivalent to the change of initial phases $\varphi_{n}(n=1 \div 8)$. Since $\Omega \ll \omega$ and $\Delta k \ll k$, two initial phases can be nullified by changing the time zero point, and four more phases (or their linear combinations) by shifting the coordinate origin.

The projections of the light pressure force $\mathbf{F}$ acting on the atom along the axes $O x$ and $O y$ equal $[1,25]$

$F_{x}=\left(\varrho_{12} \mathbf{d}_{21}+\varrho_{21} \mathbf{d}_{12}\right) \frac{\partial \mathbf{E}}{\partial x}$,

$F_{y}=\left(\varrho_{12} \mathbf{d}_{21}+\varrho_{21} \mathbf{d}_{12}\right) \frac{\partial \mathbf{E}}{\partial y}$,

where $\mathbf{d}_{i j}(i, j=1,2)$ are the matrix elements of the dipole moment, and $\varrho_{i j}$ the elements of the density matrix $\varrho$. The motion of the atom under the action of this force is described by the second Newton law

$\ddot{\mathbf{r}}=\mathbf{F} / m$,

where $m$ is the atomic mass, and $\mathbf{r}=(x, y)$ the radius vector of the atom. The density matrix is determined, by using the probability amplitudes $c_{1}$ and $c_{2}$ for the atom to be in the ground, $|1\rangle$, and excited, $|2\rangle$, states, respectively:

$\varrho_{12}=c_{1} c_{2}^{*} e^{i \omega_{0} t}, \quad \varrho_{21}=c_{2} c_{1}^{*} e^{-i \omega_{0} t}$.

The state vector of the atom

$|\psi\rangle=c_{1}|1\rangle+c_{2} e^{-i \omega_{0} t}|2\rangle$

is found from the Schrödinger equation

$i \hbar \frac{d}{d t}|\psi\rangle=H|\psi\rangle$

with the use of the Monte Carlo modeling method, in which a possibility of spontaneous light emission by the atom is taken into account [22]. Unlike computations on the basis of the density matrix, the Monte Carlo method of calculations makes it possible to trace the trajectory of motion for a separate atom.

\section{Modeling the Atomic State Vector within the Monte Carlo Method}

The Monte Carlo method for modeling the vector of state [22] is a procedure of numerical solution of the Schrödinger equation (28), which makes allowance for the possibility of a spontaneous light emission by the atom. The latter phenomenon is described by the relaxation term

$H_{\text {rel }}=-\frac{i \hbar}{2} \gamma|2\rangle\langle 2|$,

where $\gamma$ is the rate of atomic transition into the state $|1\rangle$, in the Hamiltonian

$H=H_{0}+H_{\text {int }}+H_{\text {rel }}$.

The other terms in the Hamiltonian are:

$H_{0}=\hbar \omega_{0}|2\rangle\langle 2|$,

which describes the atom in the absence of the field and relaxation, and

$H_{\text {int }}=-\mathbf{d}_{12}|1\rangle\left\langle 2\left|\mathbf{E}(t)-\mathbf{d}_{21}\right| 2\right\rangle\langle 1| \mathbf{E}(t)$,

which is responsible for the interaction between the atom and the field.

Hamiltonian (30) is non-Hermitian. Therefore, when simulating the state vector using the Monte Carlo method [22] and integrating the Schrödinger equation (28), the state vector (27) has to be renormalized after each small time step. The deviation of the state vector length from unity is used in this case to simulate the process of spontaneous photon emission: with the growth of this deviation, the probability of the spontaneous emission increases and gives rise to a quantum jump of the atom from the excited state $|2\rangle$ into the ground state $|1\rangle$. Here, we use the Monte Carlo method of the first accuracy order, which was described in work [22]. The methods of the second and fourth accuracy orders are described in work [26].

Let the atom be described by the state vector $|\psi(t)\rangle$ at the time moment $t$. The state vector $|\psi(t+\Delta t)\rangle$ at the time moment $t+\Delta t$ is determined in two stages.

(i) By integrating the Schrödinger equation (28), we obtain that, after a rather short time interval $\Delta t$, the state vector equals

$\left|\psi^{(1)}(t+\Delta t)\right\rangle=\left(1-\frac{i \Delta t}{\hbar} H\right)|\psi(t)\rangle$

ISSN 2071-0194. Ukr. J. Phys. 2019. Vol. 64, No. 2 
and its norm is

$\left\langle\psi^{(1)}(t+\Delta t) \mid \psi^{(1)}(t+\Delta t)\right\rangle=1-\Delta P$,

where

$\Delta P=\frac{i \Delta t}{\hbar}\left\langle\psi(t)\left|H-H^{+}\right| \psi(t)\right\rangle=\left.\gamma|\Delta t| c_{2}\right|^{2}$.

(ii) At the second stage, we examine if there was a quantum jump, which accompanied the spontaneous emission event, within the integration time interval $\Delta t$. For this purpose, we compare $\Delta P$ with the value $\epsilon$ of a random variable that is uniformly distributed within the interval $[0,1]$. If $\epsilon<\Delta P$, the quantum jump is assumed to occur. Hence, the atom transits into the state $|1\rangle$, i.e. its state vector becomes equal to $|1\rangle$ :

$|\psi(t+\Delta t)\rangle=|1\rangle \quad(\epsilon<\Delta P)$.

Nevertheless, in most cases, $\epsilon>\Delta P$, because $\Delta P \ll$ $\ll 1$, and the jump does not occur. In this case, the state vector (33) obtained at the first stage should be renormalized:

$|\psi(t+\Delta t)\rangle \rightarrow \frac{\left|\psi^{(1)}(t+\Delta t)\right\rangle}{\sqrt{1-\Delta P}} \quad(\epsilon>\Delta P)$.

The photon propagation direction at a spontaneous emission is also simulated, by using the Monte Carlo method. In this case, the propagation directions along the positive and negative directions of the axes $O x$ and $O y$ are regarded as equally probable (see details in Section 5).

Equation (33) gives a formal solution of the Schrödinger equation. At the same time, when calculating the state vector, it is more convenient to use the equations for the probability amplitudes $c_{1}$ and $c_{2}$ of the population of states $|1\rangle$ and $|2\rangle$. Having determined them, we obtain the state vector by formula (27). The equations for the amplitudes follow from the Schrödinger equation (28) and are equivalent to it. Let us derive those equations.

The substitution of Eqs. (27) and (30) into Eq. (28) results in

$i \hbar \frac{d}{d t} c_{1}=-\mathbf{d}_{12} \mathbf{E} c_{2} e^{-i \omega_{0} t}$,

$i \hbar \frac{d}{d t} c_{2}=-\mathbf{d}_{21} \mathbf{E} c_{1} e^{i \omega_{0} t}-\frac{1}{2} \gamma c_{2}$.

Then, in the rotating-wave approximation (by neglecting the rapidly oscillating terms proportional to

ISSN 2071-0194. Ukr. J. Phys. 2019. Vol. 64, No. 2 $\left.e^{ \pm 2 i \omega_{0} t}\right)[27]$ and taking into account that

$\mathbf{E}=\sum_{n=1}^{8} \mathbf{E}_{n}$

we obtain

$\frac{d}{d t} c_{1}=-\frac{i}{2} \Omega_{0} \sum_{n=1}^{4} e^{(-1)^{n} i k_{n} x-i \delta_{n} t+i \varphi_{n}} c_{2}-$

$-\frac{i}{2} \Omega_{0} \sum_{n=5}^{8} e^{(-1)^{n} i k_{n} y-i \delta_{n} t+i \varphi_{n}} c_{2}$

$\frac{d}{d t} c_{2}=-\frac{i}{2} \Omega_{0}^{*} \sum_{n=1}^{4} e^{(-1)^{n+1} i k_{n} x+i \delta_{n} t-i \varphi_{n}} c_{1}-$

$-\frac{i}{2} \Omega_{0}^{*} \sum_{n=5}^{8} e^{(-1)^{n+1} i k_{n} y+i \delta_{n} t-i \varphi_{n}} c_{1}-\frac{1}{2} \gamma c_{2}$,

where the Rabi frequency of monochromatic waves

$\Omega_{0}=-\mathbf{d}_{12} \mathbf{e} E_{0} / \hbar$

is introduced. Without loss of generality, this quantity can be regarded as real-valued [27].

Knowing the probability amplitudes $c_{1}$ and $c_{2}$ and using Eqs. (23), (24), and (26), we can calculate the light pressure force acting on the atom. Let us average the force expressions (23) and (24) over the time within an interval that significantly exceeds the period of rapid oscillations $\approx 2 \pi / \omega_{0}$ and, simultaneously, is rather short for the average force of light pressure to be practically independent of the averaging time interval. Then we find

$F_{x}=\hbar \sum_{n=1}^{4}(-1)^{n+1} k_{n} \operatorname{Im}\left[c_{1} c_{2}^{*} \Omega_{n}^{*} e^{i \delta_{n} t-i \varphi_{n}} \times\right.$

$\left.\times e^{i(-1)^{n+1} k_{n} x}\right]\left(\left|c_{1}\right|^{2}+\left|c_{2}\right|^{2}\right)^{-1}$.

$F_{y}=\hbar \sum_{n=5}^{8}(-1)^{n+1} k_{n} \operatorname{Im}\left[c_{1} c_{2}^{*} \Omega_{n}^{*} e^{i \delta_{n} t-i \varphi_{n}} \times\right.$

$\left.\times e^{i(-1)^{n+1} k_{n} y}\right]\left(\left|c_{1}\right|^{2}+\left|c_{2}\right|^{2}\right)^{-1}$.

Now, we can describe the motion of the atom, by simultaneously integrating Eqs. (25), (41), and (42) with regard for expressions (44) and (45) for the projections $F_{x}$ and $F_{y}$ of the light pressure force on the abscissa and ordinate axes, respectively.

The normalization in Eqs. (44) and (45) is required, when combining the integration of the Schrödinger equation and the equations of motion with the use of the fourth-order accuracy method with the Monte Carlo wave-function method of the first order. It is so because intermediate time points are used at the integration. 


\section{Numerical Calculation Procedure}

The motion of the atom was simulated by simultaneously solving Newton's equation (25) with the force described by components (44) and (45) and Eqs. (41) and (42) for the probability amplitudes of atomic states. In addition, we simulated fluctuations of the atomic momentum owing to such phenomena as the spontaneous light emission, light absorption, and induced light emission by the atom. In our calculations, for simplicity, we assumed that the spontaneous emission of a photon by the atom led to the change in the atomic momentum by $\hbar k$ with the identical probabilities in the positive and negative directions of the axes $O x$ and $O y$.

The stochastic character of the spontaneous light emission by the atom leads to its diffusion in the momentum space, this is the so-called "momentum diffusion". In the low-intensity laser radiation field, when the population of the excited state is insignificant, the light pressure force and the momentum diffusion coefficient are equal to the sum of corresponding quantities for each of the counter-propagating waves [28]. We used this approximation earlier to simulate a fluctuative change of the atomic momentum in the field of counter-propagating low-intensity light pulses [18] and to estimate a variation of the atomic momentum in the field of counter-propagating bichromatic [19], stochastic [20,29], and frequency-modulated light waves [21].

The change of the atomic momentum at the photon emission owing to the recoil effect was taken into account, by selecting the photon emission direction as follows. The interval [0,1] was divided into four identical subintervals. Each of the latter corresponded to the photon emission along the positive or negative direction of the $O x$ or $O y$ axis. At the same time, the determination of the fluctuating change of the atomic momentum due to the light absorption and induced emission required an additional attention.

As an example, let us consider how the atomic momentum fluctuates in the field of a running monochromatic wave. Let $\theta$ denote the angle between the photon spontaneous emission direction and the axis $O x$, along which the light wave propagates, and let $\left\langle N_{s}\right\rangle$ be the average number of spontaneously emitted photons. Then, assuming the photon scattering to be a completely random process, we obtain the following formula for the square of the momentum change av- eraged over an atomic ensemble [25]:

$\left\langle\Delta p_{x}^{2}\right\rangle=\left\langle\Delta p_{0 x}^{2}\right\rangle+\hbar^{2} k^{2}\left\langle N_{s}\right\rangle+\hbar^{2} k^{2}\left\langle\cos ^{2} \theta\right\rangle\left\langle N_{s}\right\rangle$.

The first term on the right-hand side is associated with the initial momentum distribution of atoms in the ensemble, the second one with the induced absorption and radiation processes, and the third one with fluctuations of the momentum at the spontaneous photon emission. Equation (46) is basic for the computer simulation of the momentum diffusion in the field of a running wave. According to this equation, one random change of the atomic momentum owing to the spontaneous light emission occurs per one change of the atomic momentum by $\pm \hbar k$ owing to induced processes. This algorithm of taking the momentum diffusion of atoms into account is valid in the case of weak fields, $\Omega_{0} \lesssim \gamma$. If the intensity of counter-propagating waves is high, the momentum diffusion in the induced processes can be taken into account in the same way, but the final results will possess an estimation character. Since we consider the motion of atoms in the field of perpendicularly intersecting waves with the same intensity, the averaged (over several modulation periods) fluctuating (owing to the induced processes) changes of the squares of the $x$ and $y$ components of the atomic momentum should expectedly be the same.

After every integration step $\Delta t$ in Eqs. (25), (41), and (42), we should determine whether a quantum jump occurred or not. If did not, the state vector is renormalized. If did, atom's velocity changes by

$\Delta v_{x}=\hbar k \operatorname{sgn}\left(\epsilon_{1}-0.5\right) \frac{1+\operatorname{sgn}\left(\epsilon_{2}-0.5\right)}{2 m}+$
$+\hbar k \operatorname{sgn}\left(\epsilon_{3}-0.5\right) \frac{1+\operatorname{sgn}\left(\epsilon_{4}-0.5\right)}{2 m}$

along the $O x$ axis and by

$\Delta v_{y}=\hbar k \operatorname{sgn}\left(\epsilon_{1}-0.5\right) \frac{1-\operatorname{sgn}\left(\epsilon_{2}-0.5\right)}{2 m}+$

$+\hbar k \operatorname{sgn}\left(\epsilon_{3}-0.5\right) \frac{1-\operatorname{sgn}\left(\epsilon_{4}-0.5\right)}{2 m}$

along the $O y$ axis, where $\epsilon_{1,2,3,4}$ are random numbers uniformly distributed within the interval $[0,1]$.

One of the terms in each of Eqs. (47) and (48) simulates the momentum fluctuation at the spontaneous photon emission along the positive and negative directions of each axis, and the other the momentum fluctuation in the same directions because of fluctuations of the induced absorption and radiation processes.

ISSN 2071-0194. Ukr. J. Phys. 2019. Vol. 64, No. 2 


\section{Results of Numerical Simulation}

The time evolution of the atomic ensemble in a bichromatic field is determined by parameters of the atomic interaction with the field and by the initial conditions. We will not complicate the analysis, by considering the distribution function of atoms over their coordinates and velocity components. Instead, we will assume that the atoms start to move from the same point, namely, the coordinate frame origin $\left(x_{0}=0, y_{0}=0\right)$, and possess the same initial velocity. The specific calculations were made for ${ }^{23} \mathrm{Na}$ atom, which can cyclically interact with the field [1]. The wavelength for the transition $3^{2} S_{1 / 2}-3^{2} P_{3 / 2}$ equals $\lambda=589.16 \mathrm{~nm}$, the spontaneous emission rate $\gamma=2 \pi \times 10 \mathrm{MHz}$, and the Doppler cooling limit for sodium atoms amounts to $T_{\mathrm{D}}=240 \mu \mathrm{K}$ [1]

Earlier, a similar research was made in the case of one standing bichromatic wave [19]. In this work, while studying the case of atomic motion in the field of counter-propagating bichromatic waves, we took parameters analogous to those used in the numerical simulation in work [19], by focusing attention on the parameters that are responsible for the formation of atomic traps and periodic patterns. In our case, a two-dimensional trap and two-dimensional spatial patterns should expectedly be formed.

The possibility of the formation of a two-dimensional trap by the field of perpendicularly intersecting bichromatic waves was evaluated by calculating the time dependences of the center-of-mass location (the mean values $x_{a v}$ and $y_{a v}$ of the atomic abscissas and ordinates, respectively) for the ensemble of atoms and the root-mean-square deviations $\Delta x$ and $\Delta y$ from the corresponding mean values. Examples of those dependences are shown in Fig. 2. The time dependences for the ordinates are not depicted, because their form is close to the corresponding dependences for the abscissas. As one can see, if the atoms start to move from the coordinate origin at the velocity $v_{0 x}=v_{0 y}=5 \mathrm{~m} / \mathrm{s}$, they are subjected to the action of a force directed toward the point $(x=0, y=0)$.

In time, fluctuations of the averaged atomic coordinates $\left(x_{a v}, y_{a v}\right)$ around the point $(x=0, y=0)$ with $\Delta x \approx 66 \mu \mathrm{m}$ and $\Delta y \approx 66 \mu \mathrm{m}$ were established. In order to make sure that those fluctuations are close to stationary ones, similar calculations were carried out for the zero initial velocity of atoms. The obtained result turned out the same (see the dashed curves in Fig. 2). The deviation turned out a bit larger than
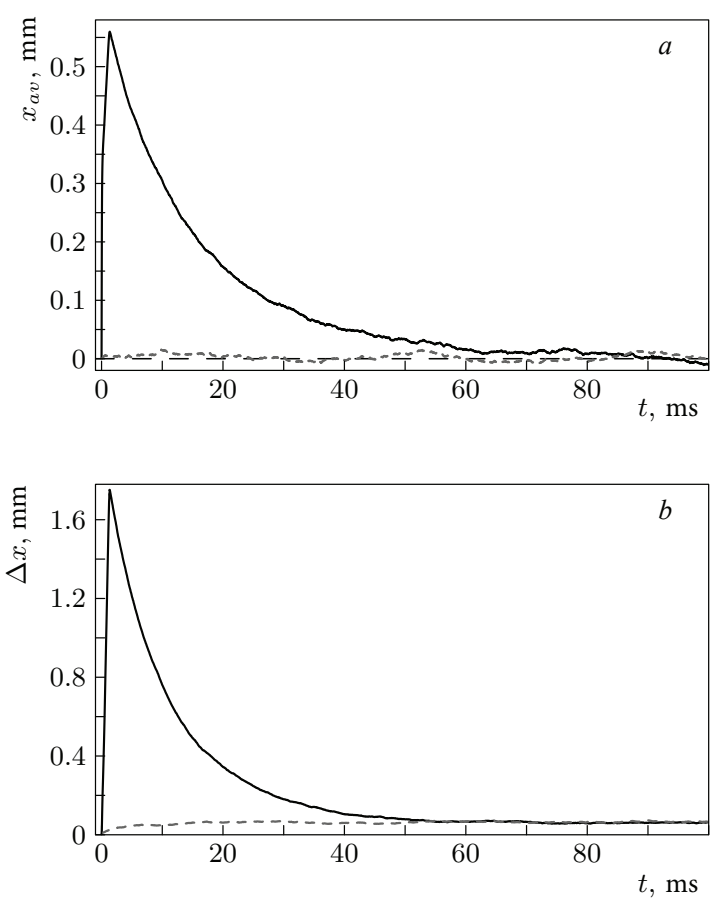

Fig. 2. Time dependences of the average coordinate $x_{a v}(a)$ and the root-mean-square deviation $\Delta x(b)$ for the abscissas of $100{ }^{23} \mathrm{Na}$ atoms at their interaction with intersecting stationary bichromatic waves. The components of the initial velocity of atoms $v_{0 x}=v_{0 y}=5 \mathrm{~m} / \mathrm{s}$ (solid curves) and $0 \mathrm{~m} / \mathrm{s}$ (dashed curves). $\Omega=2 \pi \times 200 \mathrm{MHz}, \delta=2 \pi \times 20 \mathrm{MHz}\left(\delta_{1}=\delta_{2}=\delta_{5}=\right.$ $\left.=\delta_{6}=2 \pi \times 110 \mathrm{MHz}, \delta_{3}=\delta_{4}=\delta_{7}=\delta_{8}=-2 \pi \times 90 \mathrm{MHz}\right)$, the Rabi frequencies of the waves are identical and equal to $\Omega_{0}=2 \pi \times 100 \mathrm{MHz}$, the initial phases of the waves equal zero. All atoms are in the ground state, before they start to interact with the field

the same quantity in the case of one-dimensional trap $\Delta x \approx 50 \mu \mathrm{m}$ (see Fig. 8 in work [19]). We would like to emphasize that, for the coordinate-dependent light pressure force directed to the coordinate origin to appear, the intensities of light waves have to be rather high [19].

Now, let us consider the formation of spatial atomic patterns in the field of intersecting standing bichromatic waves. We recall that the field of a standing bichromatic wave can form a one-dimensional pattern of atoms by their grouping in planes located at the distances $\frac{1}{4} \lambda+\frac{1}{2} n \lambda$ from the coordinate origin, where $n$ is an arbitrary integer [19]. Figure 3 exhibits a fragment of the spatial distribution of ${ }^{23} \mathrm{Na}$ atoms after their interaction with intersecting stationary bichromatic waves for $100 \mu \mathrm{s}$. This is a time interval suffi- 


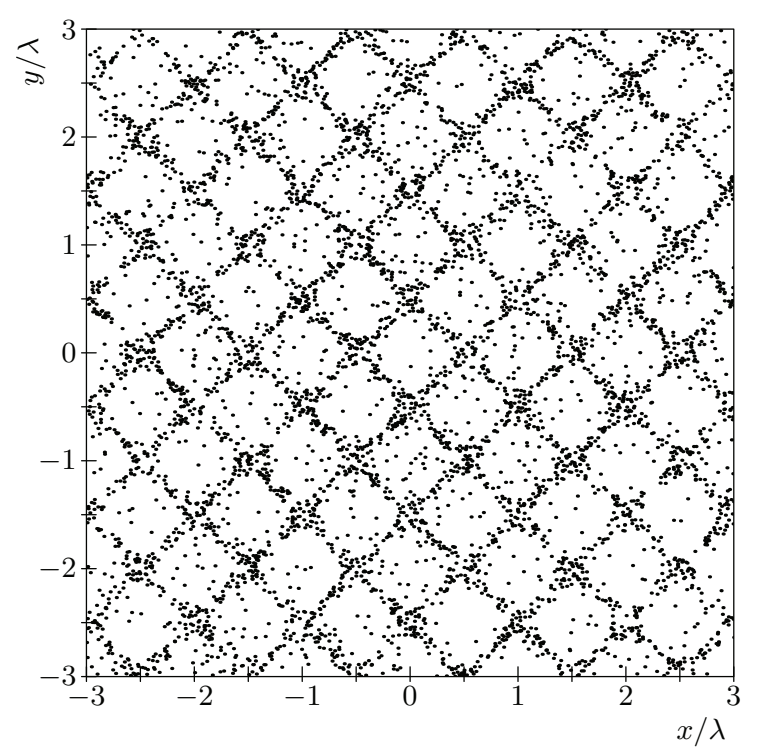

Fig. 3. Fragment of the spatial distribution of $50000{ }^{23} \mathrm{Na}$ atoms after their interaction with intersecting standing bichromatic waves for $100 \mu \mathrm{s}$. The initial velocities of atoms equal zero, $\Omega=2 \pi \times 40 \mathrm{MHz}, \delta=2 \pi \times 20 \mathrm{MHz}\left(\delta_{1}=\delta_{2}=\delta_{5}=\right.$ $\left.=\delta_{6}=2 \pi \times 120 \mathrm{MHz}, \delta_{3}=\delta_{4}=\delta_{7}=\delta_{8}=-2 \pi \times 80 \mathrm{MHz}\right)$, the Rabi frequencies of the waves are identical and equal to $\Omega_{0}=2 \pi \times 100 \mathrm{MHz}$, the initial phases of the waves equal zero

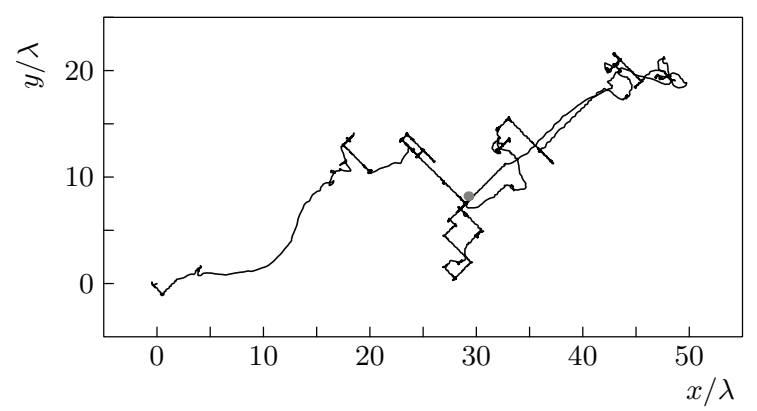

Fig. 4. Trajectory of the motion of an atom for the parameters corresponding to Fig. 3. The time of motion is $500 \mu \mathrm{s}$. The circle denotes a point corresponding to a time moment of $500 \mu \mathrm{s}$

cient for the stationary values of the components of the root-mean-square deviation of the atomic velocity from the mean values, $\Delta v_{x}=\Delta v_{y}=0.248 \mathrm{~m} / \mathrm{s}$, to be established. At the same time, at $t=100 \mu \mathrm{s}$, the rootmean-square deviations of atomic coordinates from the mean values amount to $\Delta x \approx \Delta y \approx 4.5 \mu \mathrm{m}$ and continue to grow.

The spatial distribution of atoms depicted in Fig. 3 is associated with the spatial distribution of the time- averaged energy density of the electric field, which is described by the expression

$w=8 \varepsilon_{0} E_{0}^{2}\left[\sin ^{2}(\pi x / \lambda)+\sin ^{2}(\pi y / \lambda)-1\right]^{2}$.

This formula takes into account that, for the corresponding examined distances from the coordinate origin, $x \Delta k \ll 1$ and $y \Delta k \ll 1$. From expression (49), it is clear that the energy density equals zero along the family of straight lines described by the equations

$y=x+\frac{\lambda}{2}+n_{1} \lambda$

$y=-x+\frac{\lambda}{2}+n_{2} \lambda$,

where $n_{1}$ and $n_{2}$ are integers.

As one can see from Fig. 3, most of the atoms reside near the straight lines described by Eqs. (50) and (51), i.e. in the areas, where the energy density of the electric field is low. The explanation of this phenomenon is very simple. If an atom moves in the weak field almost along the straight lines (50) and (51), the direction of its velocity either changes with a very low probability or changes after the atom has achieved a region with a strong field. But, for atoms crossing the strong-field region, the probability that the direction of their velocity will change is much higher. Thus, the distribution of atoms over their velocities has a maximum near the directions corresponding to the atomic motion along lines (50) and (51), which explains the formation of spatial pattern exhibited in Fig. 3 .

Figure 4 demonstrates a trajectory of one of the atoms. It is evident that a large part of the trajectory consists of segments directed at angles of $\pm \frac{1}{4} \pi$ with respect to the abscissa and ordinate axes, in accordance with Eqs. (50) and (51). Thus, the two-dimensional atomic pattern in Fig. 3 has a dynamic character: most of the time, the atoms move closely to the straight lines (50) and (51), and occasionally transit from one line to another.

Figure 5 illustrates the distribution of the electric field energy density in the cases $\varphi=0$ (panel $a$ ) and $\pi$ (panel $b$ ). Hence, the energy is mainly concentrated $(a)$ around the points $\left(x=n_{x} \lambda, y=n_{y} \lambda\right)$ and (b) around the points $\left(x=n_{x} \lambda+\frac{1}{2} \lambda, y=n_{y} \lambda+\frac{1}{2} \lambda\right)$, where $n_{x}$ and $n_{y}$ are arbitrary integers. The pattern formed by the atoms at $\varphi=0$ (Fig. 3) corresponds to the regions in Fig. 5, $a$ where the field is weak. If $\varphi=\pi$, the regions with the strong field are shifted by 

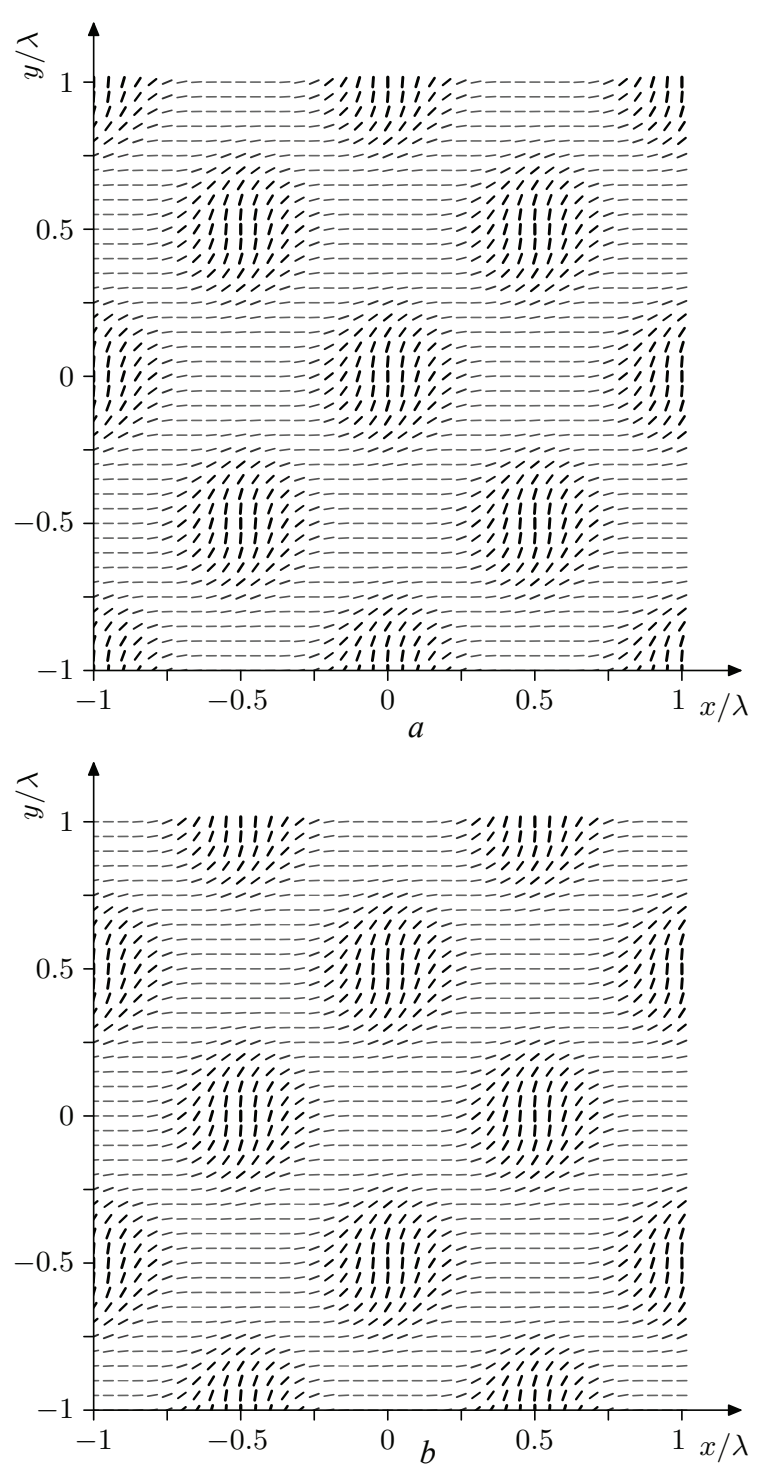

Fig. 5. Distribution of the energy density for the electric field of intersecting standing bichromatic waves in the cases (a) $\varphi=0$ (the phases of all waves are identical) and (b) $\varphi=\pi$ (the phases of the waves propagating along the ordinate axis differ by $\pi$ from the phases of waves propagating along the abscissa axis). The angle of dash inclination with respect to the abscissa axis is proportional to the field energy density

$\lambda / 2$ along the abscissa and ordinate axes, and we see again that most of the atoms reside in the regions, where the field is weak (see Fig. 6). Unlike Fig. 3, Fig. 6 demonstrates a conspicuous difference between the atomic densities at the figure center and on the figure periphery. This is a result of the slower expan-

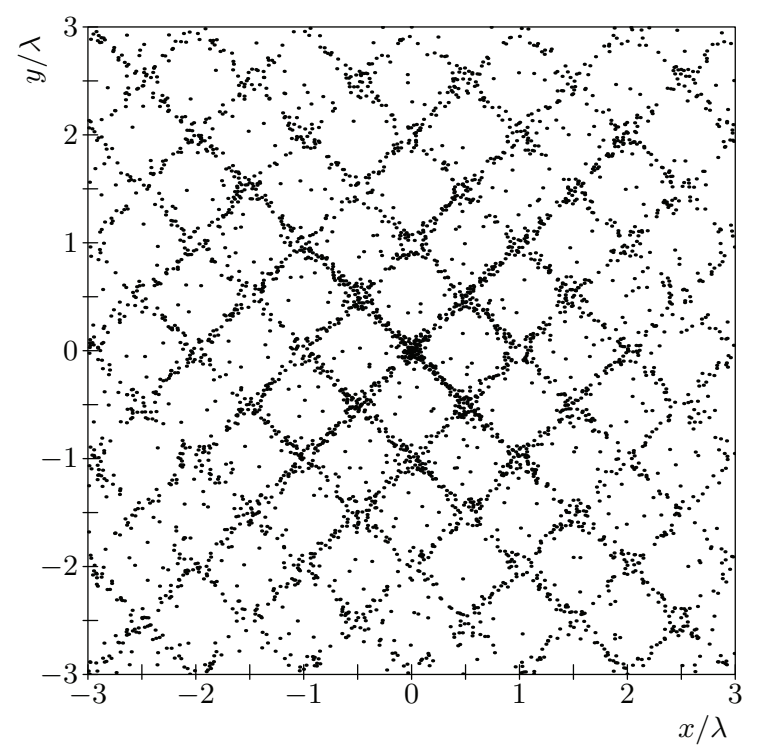

Fig. 6. Fragment of the spatial distribution of $50000{ }^{23} \mathrm{Na}$ atoms after their interaction with intersecting standing bichromatic waves for $100 \mu \mathrm{s}$. The initial velocities of atoms equal zero, $\Omega=2 \pi \times 40 \mathrm{MHz}, \delta=2 \pi \times 20 \mathrm{MHz}\left(\delta_{1}=\delta_{2}=\delta_{5}=\delta_{6}=\right.$ $\left.=2 \pi \times 120 \mathrm{MHz}, \delta_{3}=\delta_{4}=\delta_{7}=\delta_{8}=-2 \pi \times 80 \mathrm{MHz}\right)$, the Rabi frequencies of the waves are identical and equal to $\Omega_{0}=$ $=2 \pi \times 100 \mathrm{MHz}, \varphi_{1}=\varphi_{2}=\varphi_{3}=\varphi_{4}=0$, and $\varphi_{5}=\varphi_{6}=$ $=\varphi_{7}=\varphi_{8}=\pi$

sion of the atomic cloud, because the field is very low near the initial coordinates $\left(x_{0}=0, y_{0}=0\right)$ at $\varphi=\pi$. Therefore, we obtain a smaller characteristic size of the atomic cloud: $\Delta x=\Delta y=2.5 \mu \mathrm{m}$ (cf. $\Delta x=\Delta y=4.5 \mu \mathrm{m}$ at $\varphi=0$ ).

Note that, in the general case of arbitrary phase shift $\varphi$ between the intersecting waves, there are no lines in the space, along which the time-averaged energy density of the electric field

$w=4 \varepsilon_{0} E_{0}^{2}\left[2\left(\sin ^{2}(\pi x / \lambda)+\sin ^{2}(\pi y / \lambda)\right)^{2}+\right.$

$+4(\cos \varphi-1) \sin ^{2}(\pi x / \lambda) \sin ^{2}(\pi y / \lambda)+$

$\left.+\left(1-2 \sin ^{2}(\pi x / \lambda)-2 \sin ^{2}(\pi y / \lambda)\right)(1+\cos \varphi)\right]$

would be equal to zero. For example, if $\varphi=\pi / 2$, Eq. (52) reads

$w=4 \varepsilon_{0} E_{0}^{2}\left[\sin ^{2}(\pi x / \lambda)+\sin ^{2}(\pi y / \lambda)-1\right]^{2}+$

$+\sin ^{4}(\pi x / \lambda)+\sin ^{4}(\pi y / \lambda)$,

and does not equal zero at any point. At the same time, there may exist straigh intervals with a rather 
low energy density, which makes the formation of spatial patterns probable. In particular, results close to those depicted in Figs. 3 and 6 are observed at small changes in the phase shift between the intersecting waves, namely, at $\varphi=0.05 \pi$ and $0.95 \pi$, respectively.

Spatial patterns in the atomic clouds arise in the fields of intersecting standing bichromatic waves owing to a cumulative action of the waves on the atoms and cannot be interpreted by generalizing the results of the study of spatial patterns arising in the field of one standing bichromatic wave [19] to the two-dimensional case. Really, in the case of a single standing bichromatic wave, the atoms are grouped in planes at $x=\frac{1}{4} \lambda+\frac{1}{2} n \lambda$, where $n$ is an integer, for waves propagating along the abscissa axis, and $y=\frac{1}{4} \lambda+\frac{1}{2} n \lambda$ for waves propagating along the ordinate axis. By extrapolating those results onto the two-dimensional case, one should expect the grouping of atoms near the points with the coordinates $\left(x=\frac{1}{4} \lambda+\frac{1}{2} n_{x} \lambda, y=\frac{1}{4} \lambda+\frac{1}{2} n_{y} \lambda\right)$, which does not correspond to the results obtained above: a spatial pattern with a period of $\frac{\sqrt{2}}{2} \lambda$ and oriented at an angle of $\pi / 4$ with respect to the abscissa and ordinate axes.

\section{Conclusions}

The spatial distribution of atoms in the field of perpendicularly intersecting bichromatic standing waves has been studied. It is found that, the results of our previous study of the spatial distribution of atoms in the field of a stationary bichromatic wave [19] allow the parameters and the character of atomic distribution in the field of intersecting standing bichromatic waves to be estimated only partially. In particular, the parameters of the atomic distribution in a light trap created by such fields are in good agreement with the results predicted on the basis of work [19]. At the same time, the appearance of a spatial pattern cannot be predicted even roughly by extrapolating the results of work [19] obtained for the one-dimensional geometry onto the two-dimensional case: instead of the expected grouping of atoms near the points $\left(x=\frac{1}{4} \lambda+\frac{1}{2} n_{x} \lambda, y=\frac{1}{4} \lambda+\frac{1}{2} n_{y} \lambda\right)$, where $n_{x}$ and $n_{y}$ are arbitrary integers, we have their accumulation in vicinities of the straight lines corresponding to the energy minimum. For example, in the case of identical initial phases of intersecting waves, those straight lines are described by the equations $y=x+\frac{\lambda}{2}+n_{1} \lambda$ and $y=-x+\frac{\lambda}{2}+n_{2} \lambda$, where $n_{1}$ and $n_{2}$ are arbitrary integers. As a result, the atoms form a square pattern with a side of $\frac{\sqrt{2}}{2} \lambda$. This pattern has a dynamic character: an atom enters it, spends some relatively short time by moving along a pattern line, and then exits from it. After some time, the atom enter the pattern at another place, and the procedure repeats.

The publication contains the results of researches sponsored in the framework of the target complex program of fundamental researches of the National Academy of Sciences of Ukraine "Fundamental problems of the creation of new nanomaterials and nanotechnologies" (contract No. 3/18-N).

1. H. J. Metcalf, P. van der Stratten. Laser Cooling and Trapping (Springer, 1999).

2. A.M. Negriyko, V.I. Romanenko, L.P. Yatsenko. Dynamics of Atoms and Molecules in Coherent Laser Fields (Naukova Dumka, 2008) (in Ukrainian).

3. H. Metcalf. Strong optical forces on atoms in multifrequency light. Rev. Mod. Phys. 89, 041001 (2017).

4. V.S. Voitsekhovich, M.V. Danileiko, A.N. Negriiko, V.I. Romanenko, L.P. Yatsenko. Light pressure on atoms in counterpropagating amplitude-modulated waves. $Z h$. Tekhn. Fiz. 58, 1174 (1988) (in Russian).

5. A.P. Kazantsev, I.V. Krasnov. Rectification effect of a radiation force. J. Opt. Soc. Am. B 6, 2140 (1989).

6. V.S. Voitsekhovich, M.V. Danileiko, A.N. Negriiko, V.I. Romanenko, L.P. Yatsenko. Observation of a stimulated radiation pressure of amplitude-modulated light on atoms. JETP Lett. 49, 161 (1989).

7. R. Grimm, Y.B. Ovchinnikov, A.I. Sidorov, V. S. Letokhov. Observation of a strong rectified dipole force in a bichromatic standing light wave. Phys. Rev. Lett. 65, 1415 (1990).

8. Y.B. Ovchinnikov, R. Grimm, A.I. Sidorov, V.S. Letokhov. Recified dipole force in a bichromatic standing light wave. Opt. Commun. 102, 155 (1993).

9. J. Söding, R. Grimm, Y. Ovchinnikov, P. Bouyer, C. Salomon. Short-distance atomic beam deceleration with a stimulated light force. Phys. Rev. Lett. 78, 1420 (1997).

10. M.R. Williams, F. Chi, M.T. Cashen, H. Metcalf. Bichromatic force measurements using atomic beam deflections. Phys. Rev. A 61, 023408 (2000).

11. M.T. Cashen, H. Metcalf. Bichromatic force on helium. Phys. Rev. A 63, 025406 (2001).

12. C. Corder, B. Arnold, H. Metcalf. Laser cooling without spontaneous emission. Phys. Rev. Lett. 114, 043002 (2015).

13. C. Corder, B. Arnold, X. Hua, H. Metcalf. Laser cooling without spontaneous emission using the bichromatic force. J. Opt. Soc. Am. B 32, B75 (2015).

ISSN 2071-0194. Ukr. J. Phys. 2019. Vol. 64, No. 2 
14. H. Metcalf. Entropy exchange in laser cooling. Phys. Rev. $A$ 77, 061401 (2008).

15. T.G.M. Freegarde, J. Waltz, W. Hänsch. Confinement and manipulation of atoms using short laser pulses. Opt. Commun. 117, 262 (1995).

16. A. Goepfert, I. Bloch, D. Haubrich, F. Lison, R. Schütze, R. Wynands, D. Meschede. Stimulated focusing and deflection of an atomic beam using picosecond laser pulses. Phys. Rev. A 56, R3354 (1997).

17. V.I. Romanenko, L.P. Yatsenko. Theory of one-dimensional trapping of atoms by counterpropagating short pulse trains. J. Phys. B 44, 115305 (2011).

18. V. I. Romanenko, Ye.G. Udovitskaya, A.V. Romanenko, L.P. Yatsenko. Cooling and trapping of atoms and molecules by counterpropagating pulse trains. Phys. Rev. A 90, 053421 (2014).

19. V.I. Romanenko, A.V. Romanenko, L.P. Yatsenko. An optical trap for atoms on the basis of counter-propagating bichromatic light waves. Ukr. J. Phys. 61, 309 (2016).

20. V.I. Romanenko, L.P. Yatsenko. Trapping of atoms by the counter-propagating stochastic light waves. Opt. Commun. 392, 239 (2017).

21. V.I. Romanenko, N.V. Kornilovska. Atoms in the counterpropagating frequency-modulated waves: splitting, cooling, confinement. Eur. Phys. J. D 71, 229 (2017).

22. C. Mølmer, Y. Castin, J. Dalibard. Monte Carlo wavefunction method in quantum optics. J. Opt. Soc. Am. B 10, 524 (1993).

23. L. Yatsenko, H. Metcalf. Dressed-atom description of the bichromatic force. Phys. Rev. A 70, 063402 (2004).

24. A.P. Kazantsev. Acceleration of atoms by light. Zh. Éksp. Teor. Fiz. 66, 1599 (1974).
25. V.G. Minogin, V.S. Letokhov. Laser Light Pressure on Atoms (Gordon and Breach, 1987).

26. J. Steinbach, B.M. Garraway, P.L. Knight. High-order unraveling of master equations for dissipative evolution. Phys. Rev. A 51, 3302 (1995).

27. B.W. Shore. The Theory of Coherent Atomic Excitation, Vol. 1 (Wiley, 1990).

28. K. Mølmer. Limits of Doppler cooling in pulsed laser fields. Phys. Rev. Lett. 66, 2301 (1991).

29. V.I. Romanenko, O.G. Udovytska, V.M. Khodakovsky, L.P. Yatsenko. Atomic momentum diffusion in the field of counter-propagating stochastic light waves. Ukr. J. Phys. 63, 616 (2018)

Received 19.11.18.

Translated from Ukrainian by O.I. Voitenko

В.І. Романенко, Н.В. Корніловсъка,

О.Г. Удовицъка, Л.П. Яченко

ПРОСТОРОВИЙ РОЗПОДІЛ

АТОМІВ У ПОЛІ ПЕРЕХРЕСНИХ СТОЯЧИХ

БІХРОМАТИЧНИХ СВІТЛОВИХ ХВИЛЬ

$\mathrm{P}$ е $з$ ю м е

Показано, що, вибираючи належним чином відстроювання несучої частоти кожної з перехресних біхроматичних хвиль від частоти переходу в атомі, можна сформувати двовимірну пастку для атомів, якщо інтенсивність хвиль досить велика. При нульових та близьких до нуля початкових фазах хвиль, а також при $\pi$ та близькому до $\pi$ зсуві фаз між перехресними хвилями формується динамічна просторова структура з квадратних комірок зі стороною $\lambda / \sqrt{2}$. Чисельне моделювання проведено для атомів натрію. 